\title{
The dominant role of structure for solute transport in soil: experimental evidence and modelling of structure and transport in a field experiment
}

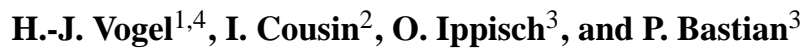 \\ ${ }^{1}$ Institute of Environmental Physics, University of Heidelberg, Germany \\ ${ }^{2}$ INRA, Unité de Science du Sol, Orléans, France \\ ${ }^{3}$ Interdisciplinary Center of Scientific Computing, University of Heidelberg, Germany \\ ${ }^{4}$ UFZ - Center for Environmental Research, Leipzig-Halle, Germany
}

Received: 1 September 2005 - Published in Hydrol. Earth Syst. Sci. Discuss.: 30 September 2005

Revised: 8 March 2006 - Accepted: 6 June 2006 - Published: 7 July 2006

\begin{abstract}
A classical transport experiment was performed in a field plot of $2.5 \mathrm{~m}^{2}$ using the dye tracer brilliant blue. The measured tracer distribution demonstrates the dominant role of the heterogeneous soil structure for solute transport. As with many other published experiments, this evidences the need of considering the macroscopic structure of soil to predict flow and transport.

We combine three different approaches to represent the relevant structure of the specific situation of our experiment: i) direct measurement, ii) statistical description of heterogeneities and iii) a conceptual model of structure formation. The structure of soil layers was directly obtained from serial sections in the field. The sub-scale heterogeneity within the soil horizons was modelled through correlated random fields with estimated correlation lengths and anisotropy. Earthworm burrows played a dominant role at the transition between the upper soil horizon and the subsoil. A model based on percolation theory is introduced that mimics the geometry of earthworm burrow systems.
\end{abstract}

The hydraulic material properties of the different structural units were obtained by direct measurements where available and by a best estimate otherwise. From the hydraulic structure, the 3-dimensional velocity field of water was calculated by solving Richards' Equation and solute transport was simulated. The simulated tracer distribution compares reasonably well with the experimental data. We conclude that a rough representation of the structure and a rough representation of the hydraulic properties might be sufficient to predict flow and transport, but both elements are definitely required.

Correspondence to: H.-J. Vogel

(hjvogel@ufz.de)

\section{Introduction}

Solute transport is important to plant uptake, chemical reactions and transformation processes, and is a key factor in controlling groundwater contamination.

Despite its fundamental importance, solute transport in soil is still far from being quantitatively understood. This means we are typically not in the position to predict solute transport with respect to the first arrival time in a given depth or even with respect to the mean velocity.

The major hurdle is the macroscopic heterogeneity of soils at various spatial scales. The consequence is that at a given length scale or a given region of interest we typically find some sort of heterogeneity which has a similar characteristic length scale. In this case, no meaningful averaging, and thus, no meaningful effective model parameters and no representative elementary volume (REV) can be found. In the following, we want to use the term "structure" for that type of heterogeneity which is of similar size as the scale of interest (i.e. size of sample) and hence, can not be represented by effective properties.

A well known phenomenon, which can be considered as a consequence of heterogeneous material properties, is "preferential flow", in which some fraction of a solute moves much faster than expected, through a small fraction of the medium. In this case, wrong expectations typically originate from the measurement of "effective" material properties assuming homogeneity in the sense of a REV while the preferential flow paths are not captured by the measurement. This phenomenon of preferential flow was first attributed to continuous macropores (e.g. Bouma, 1981; Beven and Germann, 1982) and has been evidenced for years in both laboratory and field experiments. The use of dye tracers by Flury et al. (1994) allowed a detailed study of the phenomenology of solute transport thereby revealing that preferential flow as

Published by Copernicus GmbH on behalf of the European Geosciences Union. 
a consequence of various soil structural features was the rule rather than the exception. It is not only produced by macropores but also, more generally, by the heterogeneous structure of hydraulic properties.

The common approach for modelling preferential flow is to separate different zones with different mobility but fixed volume fraction (van Genuchten and Wierenga, 1976; Gerke and van Genuchten, 1993; Jarvis, 1994). This approach adds considerable flexibility to the classical convection-dispersion model, so that experimental findings can be described quite accurately (Kätterer et al., 2001; Larsbo et al., 2005). However these models are only effective descriptions of the natural complexity, and the additional parameters (i.e. the volume fraction of zones with different mobility and the rate parameter controlling the exchange between these zones) cannot be measured independently. Therefore, only a retrospective description of a specific experiment is feasible through the fitting of the model parameters to measured breakthrough curves or spatial distributions of concentration data. Moreover, the obtained parameters may change with the initial and boundary conditions due to the changing geometry of the flow field (Clothier et al., 1995) and with the transport distance due to the heterogeneity of the material (Vanderborght et al., 2001). Hence, the usage of these models may be uncertain for conditions other than those used for calibration.

In the context of flow and transport, relevant structures are ubiquitous at a wide range of spatial scales, which has been formalised for soils using the concept of fractal geometry (Baveye and Boast, 1998) or the approach of discrete or continuous hierarchy (Cushman, 1990). Vogel and Roth (2003) proposed the "scaleway" as a discrete hierarchical approach for modelling flow and transport, which is based on the explicit consideration of the macro structure while microscopic heterogeneities are averaged and replaced by effective descriptions. The major drawback of this approach is the fact that a full three-dimensional representation of the relevant structure is required. Given the heterogeneity of natural soil, there seems to be no loophole out of this dilemma, if we would like to come up with a reliable prediction.

In this paper we intend to present three different approaches to incorporating the relevant 3-D-structure of a given soil for modelling flow and transport, and we discuss meanings of the term "relevant" in this context.

The first approach, which will be referred to as the "explicit" approach, is based on the direct measurement of the structure using appropriate instruments. This can be done using destructive methods as e.g. serial sectioning. Today, however, there are powerful instruments available for the noninvasive measurement of structure using the concept of tomography. At the small scale $\mathrm{x}$-ray tomography is used to identify the structure of macropores and zones of contrasting bulk densities (Hopmans et al., 1994; Wildenschild et al., 2002). On larger scales geophysical tools might be applied, such as geoelectric (e.g. Puvance and Andricevic, 2000) or georadar (e.g. Kowalsky et al., 2004) techniques.
The second approach is a "statistical" approach. It is based on limited information on relevant structural properties such as the variance and the correlation scale of material properties. Given this information, a continuous representation of the structure can be represented by equivalent random fields. This approach is typically used in the field of stochastic continuum theory (Dagan, 1986; Gelhar, 1986).

The third approach is termed the "genetic" approach. It uses the available knowledge on the processes of structure formation to generate the structure itself. This approach is especially attractive for structural units which are highly relevant but sparse and/or highly anisotropic, so that the first approach is not adequate and the second is insufficient in terms of spatial resolution. Examples are fissures or earthworm burrows.

We apply these different strategies to predict the 3-D spatial distribution of a dye tracer, which was measured in a field experiment at steady state flow conditions. The structure of the soil horizons is directly measured by photography (explicit approach), the sub-scale heterogeneity is modelled through estimated correlation lengths within the soil horizons (statistical approach), and the macroporous structure of earthworm burrows connecting the topsoil and the subsoil is modelled based on a few typical properties of the burrow system of anecic earthworms (genetic approach). Ideally, the hydraulic properties for each structural unit are measured directly. Where this was not possible we applied a best estimate. Then, based on the continuous 3-dimensional representation of the structure of the experimental domain and the hydraulic properties of the individual structural units, we simulated steady state water flow using Richards' equation. Subsequently, the resulting velocity field was used to simulate solute transport assuming convection-dispersion type of transport at the small scale. Finally, the simulated dye-tracer distribution is directly compared to experimental data. The simulation is considered to be a prediction of solute transport, in the sense that no result of the transport experiment is used for model calibration. It is based on a rather rough description of the spatial heterogeneity and a rather rough approximation of the hydraulic properties. One aim of this study was to evaluate how far we can get in face of such rough approximations - a typical situation in field experiments - by combining structural information with material properties.

\section{Experiment}

The experiment was conducted in the Beauce region, in France on a $80 \mathrm{~cm}$ deep loamy-clay soil classified as a haplic Calcisol (FAO-UNESCO, 1975). It is organised in 3 horizons (Ould Mohammed and Bruand, 1994): from 0 to $35 \mathrm{~cm}$, a formerly ploughed horizon, $A$, with a plough-pan at $35 \mathrm{~cm}$ depth; from 35 to $60 \mathrm{~cm}$, a structured $B$ horizon, with less organic matter; and from 60 to $80 \mathrm{~cm}$, a cryoturbated calcareous $C$ horizon including calcareous stones. At the date 
of the experiment, in April 2000, the soil was covered with rape. During the 5 years prior to our experiment the soil was treated with minimum tillage restricted to the upper $10 \mathrm{~cm}$ using a grubber. Before, the soil was annually ploughed to a depth of $35 \mathrm{~cm}$. Due to the change in tillage practice, the upper $A$ horizon was partitioned into two parts: a loose, crumbly structured surface layer, $A_{1}$, down to $10 \mathrm{~cm}$, and a lower part, $A_{2}$, from 10 to $35 \mathrm{~cm}$ where the soil was markedly more compact with a polyhedric structure and vertical earthworm burrows.

The infiltration experiment was performed on a plot of $1.6 \times 1.6 \mathrm{~m}$. Water and dye tracer were supplied using an automated sprinkler device with 5 nozzles spaced $33 \mathrm{~cm}$ apart (Kasteel et al., 2002). The distance between the sprinkler and the soil surface was adjusted to $36 \mathrm{~cm}$ to ensure a uniform irrigation of the soil surface. The experiment was performed inside a tent to avoid any disturbance by wind and natural rainfall. The kinetic energy of the small water droplets produced by the nozzles was small enough to avoid modifications of the soil surface. The applied steady state infiltration rate was adjusted to $13 \mathrm{~mm} \mathrm{~h}^{-1}$ to avoid the formation of puddles at the soil surface. To stain the flow paths, we used the food dye brilliant blue FCF which is frequently used because of the supposedly low interaction of the anionic molecule with the soil matrix (Flury and Flühler, 1995). However, it was found that the retardation of brilliant blue might be significant depending on the ionic strength and the soil type (Ketelsen and Meyer-Windel, 1999; Germàn-Heins and Flury, 2000; Kasteel et al., 2002). Consequently we interpret the stained part of the soil as minimum travel distance. The experiment consisted of 3 consecutive periods while the flow rate was kept constant:

1. The soil profile was irrigated for $16 \mathrm{~h}$ by $210 \mathrm{~mm}$ of tracer-free water. After this period we assumed steady state flow in the upper part of the soil profile.

2. During the second period, $19 \mathrm{~mm}$ of the dye tracer $(6 \mathrm{~g} / \mathrm{l}$ brilliant blue) was applied as an extended pulse.

3. Finally, $55 \mathrm{~mm}$ of tracer-free water were applied to translocate the dye pulse.

After the infiltration experiment, a vertical soil pit $(140 \mathrm{~cm}$ width, $90 \mathrm{~cm}$ depth) was excavated within the irrigated area. This vertical section was photographed together with a grey frame containing a metric colour scale (Fig. 1). Then, to produce serial sections, a slice of $4 \mathrm{~cm}$ was removed from the pit using a knife and another image was taken. This procedure was repeated 23 times to produce a set of 24 digital serial sections with a separation of $4 \mathrm{~cm}$. The individual images cover an area of about $100 \mathrm{~cm}$ width and $70 \mathrm{~cm}$ height at a resolution of $960 \times 745$ pixel with a pixel size of $1 \mathrm{~mm}^{2}$.

At a close distance from the experiment, undisturbed soil cores $(16.2 \mathrm{~cm}$ diameter, $10 \mathrm{~cm}$ height) were taken from the $A_{1}$ and $B$ horizon. These samples were used for classical multistep-outflow experiments (Hopmans et al., 2002) to determine the hydraulic properties of the different horizons. The sample taken from the compacted $A_{2}$ horizon had to be rejected because of a significant disturbance of the structure.

\section{Image analysis}

\subsection{Extraction of structural units}

First the serial images were corrected for distortion using the spatial coordinates of the edges which are clearly identified by the fixed frame. Then, the main horizons $\left(A_{1}, A_{2}, B\right)$ could be identified based on different colours. After combining the serial sections we got the 3-dimensional structure of the soil horizons at a resolution of $1 \mathrm{~mm} \times 40 \mathrm{~mm} \times 1 \mathrm{~mm}$ in the $\mathrm{x}, \mathrm{y}$, and $\mathrm{z}$ direction, respectively (Fig. 1). Earthworm burrows were clearly detected as an additional structural unit especially in the compacted horizon $A_{2}$. Evidently, the resolution of the serial sections was far too low to measure the 3-dimensional geometry of such burrows explicitly.

\subsection{Distribution of dye tracer}

The 3-dimensional distribution of the dye tracer was obtained at the same resolution as the soil horizons. The images were recorded in RGB format with brightness values in the range $0-255$, and we used the red $(r)$ and the green $(g)$ channel to discriminate blue stained areas without distinguishing different concentrations. A voxel was considered to be stained if

$\frac{r}{g}<1.45$ and $r+g<[\bar{r}+60]$,

where $\bar{r}$ is the mean red value of the image. This rule was applied to each image individually. Because the $r / g$ ratio is insensitive to brightness, and the threshold value is adjusted for mean image brightness, differences in illumination between images during the excavation and photography period are accounted for. No further image correction was considered necessary.

\section{Modelling soil structure}

\subsection{Soil horizons - explicit description}

A description of the soil horizons was obtained from the serial sections described above, meaning the boundaries between the different horizons which were found to be not exactly horizontal, are directly measured by the serial sections. Together with the experimental determination of the hydraulic properties, a complete 3-dimensional description of the hydraulic conditions should be possible. Such an approach would have to rely on the assumption of macroscopic homogeneity of the different horizons, specifically, that the soil cores used to measure the hydraulic properties include a 


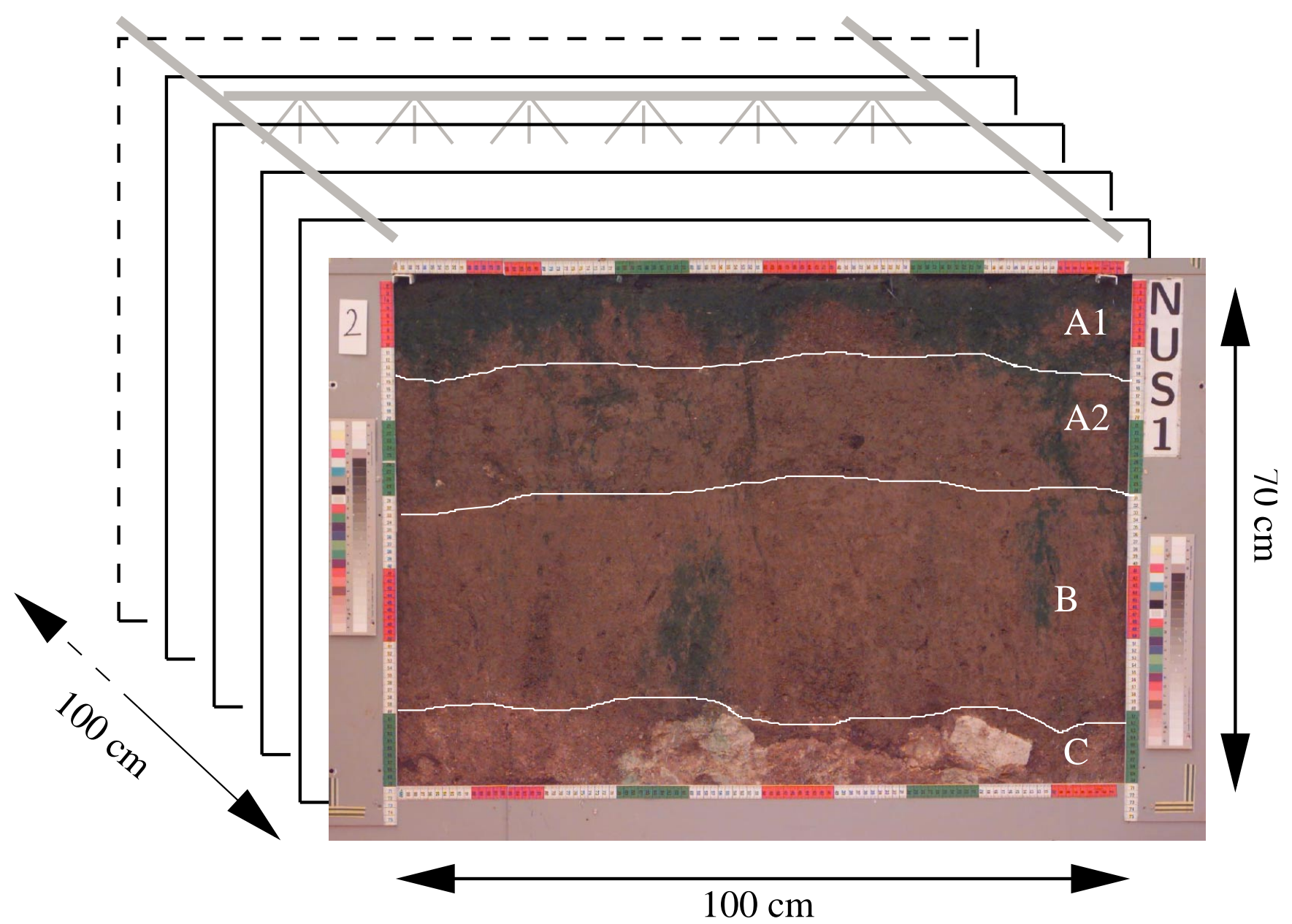

Fig. 1. Setup of the experiment: After the application of the dye tracer, the experimental domain $\left(1.0 \times 1.0 \times 0.7 \mathrm{~m}^{3}\right)$ was explored by 24 serial sections. Notice the irregular distribution of the dye.

representative elementary volume (REV). In our experiment, this requirement was definitely violated by the macropores within the $A_{2}$ horizon formed by earthworms. Moreover, the $A_{1}$ and the $B$ horizon exhibited some mesoscopic heterogeneities which were smaller but close to the size of the cores. In the $A_{1}$ horizon there were aggregates and clods resulting from tillage; the $B$ horizon had regions of variable bulk density due to variable biological activity (roots, earthworms). Because the characteristic size of these structural features was not much smaller than the thickness of the soil horizons, the assumption of macroscopic homogeneity was critical also in the $A_{1}$ and $B$ horizon. For a quantitative understanding of flow and transport, all these mesoscopic heterogeneities might be significant. On the other hand, no direct measurement of the mesoscopic structure is available, which could be used in a numerical model to predict flow and transport. In the following we use a classical statistical approach to represent mesoscopic heterogeneities in the $A_{1}$ and the $B$ horizon, and a new genetic approach to represent the macroporous structure within the $A_{2}$ horizon.

\subsection{Mesoscopic heterogeneity - statistical approach}

In the $A_{1}$ and the $B$ horizons a mesoscale heterogeneity was introduced by varying the measured hydraulic properties $h^{*}(\theta)$ and $K^{*}(\theta)$ where $h$ is the soil water potential, $\theta$ the volumetric water content, and $K$ the hydraulic conductivity. We used a scaling factor $c$ according to (Roth, 1995),

$c=\frac{h^{*}(\theta)}{h(\theta)}$,

so that the actual water characteristic at a given location, $h(\theta)$, is related to the measured reference properties through $c$. For the hydraulic conductivity we use

$c^{2}=\frac{K(\theta)}{K^{*}(\theta)}$.

This approach implies similarity of the region with different hydraulic properties according to Miller and Miller (1956). The scaling factor $c$ reflects a characteristic length at the pore scale such as grain size or pore radius, which is typically assumed to be $\log$-normally distributed. We considered $\log (c)$ 
to be normally distributed with expectation 0 and variance $\sigma_{c}^{2}=0.25$. We use the same scaling factor to describe both, $h(\theta)$ and $K(\theta)$, while $h(\theta)$ is linearly related to the characteristic length according to Eq. (2) and $K(\theta)$ is proportional to $c^{2}$ according to Eq. (3). The spatial correlation of $c$ is described by a Gaussian random field with predefined covariance function and related correlation lengths $\lambda_{x, y, z}$ for the three spatial directions. The random fields were generated according to Robin et al. (1993).

In the $A_{1}$ horizon we chose the mesoscopic heterogeneity to be slightly anisotropic with a preferred horizontal orientation, i.e. $\lambda_{x, y}=45 \mathrm{~mm}$ and $\lambda_{z}=30 \mathrm{~mm}$. This choice was based on field observation for the orientation of soil clods formed by tillage. In the $B$ horizon, a preferred vertical orientation was assumed with a correlation length of $\lambda_{x, y}=60 \mathrm{~mm}$ in the horizontal and $\lambda_{z}=120 \mathrm{~mm}$ in the vertical direction, which was also based on field observations.

\subsection{Macroporous structure - genetic approach}

The phenomenology of flow and transport through the compacted soil layer $A_{2}$ is dominated by the macroporous structure due to earthworm activity and roots. Direct measurements of the detailed geometry of this structural unit was not possible since the resolution of the field images is much too low, because the distance between the serial sections is too large. However, the macroporous structure cannot be ignored for a quantitative and even qualitative understanding of flow and transport in our experiment. The huge difference between the characteristic size of a structural element $\lambda$, in this case the diameter of the macropores, and the functional correlation length of the structural unit $\Lambda$, here the continuous lengths of the macropores, is a general difficulty. This is true for the measurement as well as for the spatial discretisation of structural components for modelling purposes. In both cases the resolution, i.e. the pixel size, needs to be smaller than $\lambda$ while the region of measurement should be larger than $\Lambda$. The technical difficulties to achieve this requirement increase with increasing anisotropy of the structure.

An alternative to the direct measurement of the geometry of earthworm burrow systems, is to model this geometry using the available knowledge on the way these burrow systems are created. Such an approach was recently proposed by Bastardie et al. (2002) who simulated the burrowing activity of different earthworm species based on the statistics of characteristic types of earthworm movements defined by Capowiez (2000). They used this "animal-based" model to investigate the effect of various structural aspects, such as volume, topology and orientation, on the hydraulic properties of earthworm channel systems.

Naturally, this approach cannot reproduce any details related to the multitude of different species having specific habits, which additionally might be modified by the type of substrate they are living in. Also the diameter of earthworm burrows varies considerably. For our experiment, how- ever, the exact diameter is less important with respect to the overall pattern of solute transport because, in any case, the saturated hydraulic conductivity of the macropores is very much higher than that of the surrounding soil matrix. For our application we only used some general information on the basic geometry of earthworm channels available from the literature. Field measurements of borrow systems created by the large anecic species Lumbricus terrestris, Aporrectodea spec. and Allolobophora spec. indicated a vertical main channel with an increased tendency for branching towards the top soil which leads to a funnel like structure (Lighthart et al., 1993; Jegou et al., 1999). As direct information from our field experiment, we estimated the number density of continuous earthworm burrows in the horizontal plane to be $9 \mathrm{~m}^{-2}$ within the compacted $A_{2}$ horizon. This was done through visual inspection during the excavation of the soil.

To model a single macropore we used the concepts of percolation theory (Stauffer, 1985). We started from an uncorrelated field of random numbers equally distributed in $[0,255]$ on a cubic voxel grid. The horizontal dimensions (256 voxel) of this field corresponds to the area attributed to one macropore (1/9 of the total area) and the vertical dimension corresponds to the depth of the compacted horizon $A_{2}$. Then, the random grey field was binarized just above the percolation threshold so that a percolation cluster with at least one continuous path in the vertical direction is produced and we deleted all voxels that did not belong to this continuous path to isolate the backbone of the percolation cluster. Then the most efficient path was determined through numerical simulation of transport through the backbone using a particle tracking algorithm: After reducing the backbone to its skeleton (i.e. a path of 1 voxel thickness) a number of particles were introduced at a randomly chosen top end of the backbone. Then, for each particle a random walk through the backbone was calculated to obtain the shortest path and "critical neck" of the backbone. The latter is defined as the location within the backbone where most of the particles have passed. Below this point only the shortest path was considered while above that point all paths inside the backbone were conserved. Hence, a funnel like macropore was generated reflecting some geometric properties of natural earthworm burrows. Finally the skeleton of the simulated macropore was dilated by a spherical structuring element to adjust the radius of the channels to a constant value of $7.5 \mathrm{~mm}$.

This approach for modelling the macroporous structure of the compacted soil layer also reflects the tendency of roots to pass through such layers along earthworm channels (Sveistrup et al., 1997) acting as preferential flow paths (Jarvis et al., 1987). Once a root has "found" an earthworm channel it follows that channel to reach the more favourable layers below the compacted zone. Figure 2 shows different realizations of generated macropores.

Figure 3 shows the entire 3-D representation of the modelled domain including iso-surfaces of the scaling factors 

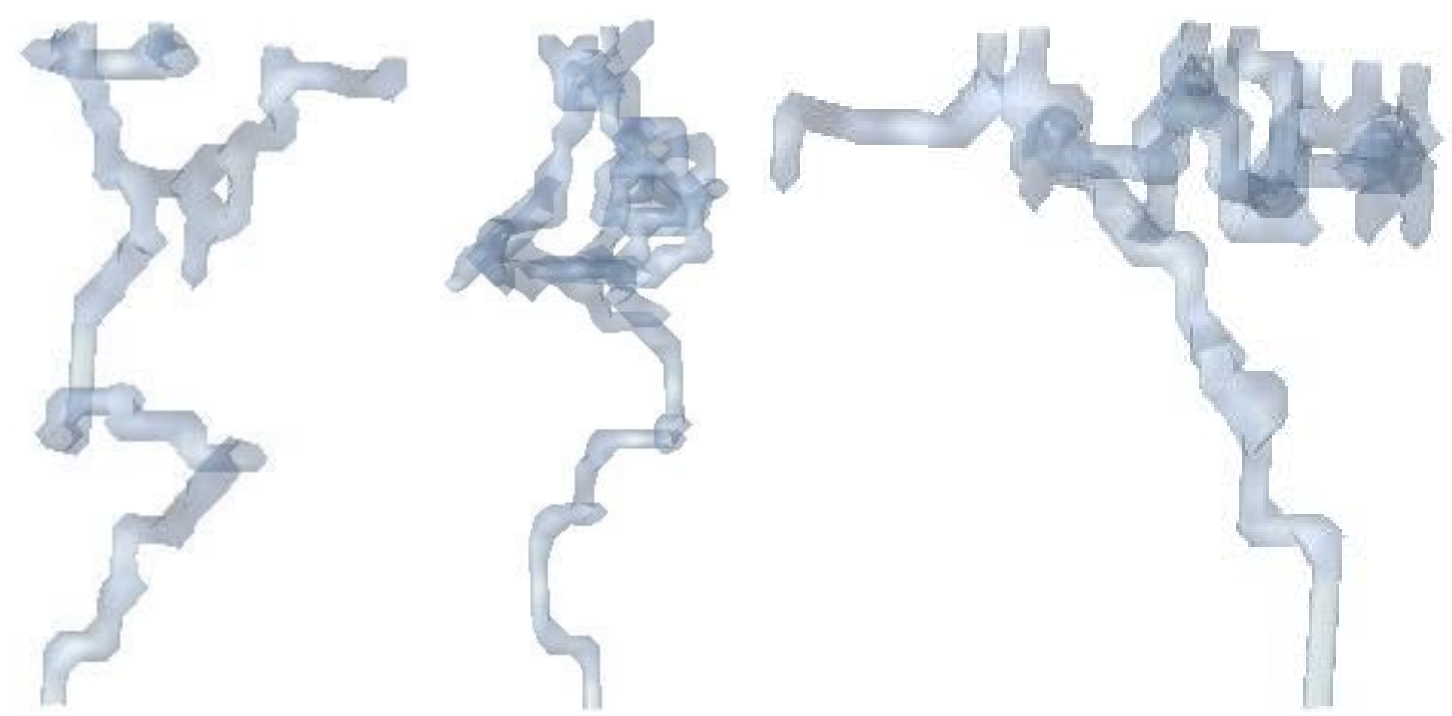

Fig. 2. Three realizations of earthworm burrows using a genetic model.

describing the mesoscopic heterogeneity in the $A_{1}$ and $B$ horizon and the earthworm burrows in the $A_{2}$ horizon.

\section{Numerical simulation of flow and transport}

\subsection{Water flow}

Based on the complete three-dimensional representation of the structure within the experimental domain (Fig. 3), we calculated the heterogeneous velocity field during the infiltration experiment using Richards' equation:

$\frac{\partial}{\partial t} \theta-\nabla \cdot\left[K(\theta)\left[\nabla h-\rho_{w} g\right]\right]=0$

where $\rho_{w}$ is the density of water and $g$ acceleration due to gravity. To solve this equation the hydraulic properties $h(\theta)$ and $K(\theta)$ are required for each location. This can be obtained, given the measured structure of the different horizons and assuming macroscopic homogeneity for each horizon with or without mesoscopic heterogeneity. However, it is not obvious how to handle the macroporous structure of the $A_{2}$ horizon. Clearly, Richards' equation does not apply to the flow of free water in macropores. Actually, the problem of coupling flow in macropores with flow in the soil matrix within a single model framework is still not solved. As an approximation, we considered the macropores to be a very coarse porous medium having a low air entry value and a high conductivity as was done by Gerke and van Genuchten (1993).

The hydraulic properties for each structural unit - soil horizons and macropores - are described using a modified van Genuchten model according to Vogel et al. (2001). This model introduces an explicit air entry pressure $h_{e}<0$ to the classical model of van Genuchten (1980) and is given by

$S_{e}=\left\{\begin{array}{ll}\frac{1}{S_{c}}\left[1+[-\alpha h]^{n}\right]^{-m} & : h<h_{e} \\ 1 & : h \geq h_{e}\end{array}\right.$,

with the effective water saturation

$S_{e}=\frac{\theta-\theta_{r}}{\theta_{s}-\theta_{r}}$.

$\theta_{s}$ and $\theta_{r}$ are the saturated and the residual water content respectively, $n$ and $\alpha$ are the empirical van Genuchten parameters, and $m=1-1 / n . S_{c}=\left[1+\left[-\alpha h_{e}\right]^{n}\right]^{-m}$ denotes the saturation at the air entry point using the classical van Genuchten model.

This formulation was introduced to avoid an unrealistically steep decline of the hydraulic conductivity close to water saturation when using the Mualem-model to derive the hydraulic conductivity function (Mualem, 1976). It can be shown, that such an unrealistic shape of the conductivity function has to be expected for $n<2$ and that it hampers the numerical solution of Richards' equation considerably. For a detailed discussion we refer to Ippisch et al. (2005, 2006).

The relative hydraulic conductivity is then given by

$K_{r}=\left\{\begin{array}{ll}S_{e}^{\tau}\left[\frac{1-\left(1-\left(S_{e} S_{c}\right)^{1 / m}\right)^{m}}{1-\left(1-\left(S_{c}\right)^{1 / m}\right)^{m}}\right]^{2} & : \quad S_{e}<S_{c} \\ 1 & : \quad S_{e} \geq S_{c}\end{array}\right.$,

and the hydraulic conductivity function as

$K\left(S_{e}\right)=K_{S} K_{r}\left(S_{e}\right)$,

where $K_{S}$ is the saturated hydraulic conductivity and $\tau$ is an empirical parameter for tortuosity.

For the $A_{1}$ and the $B$ horizons, the hydraulic parameters were determined through inverse modeling based on 


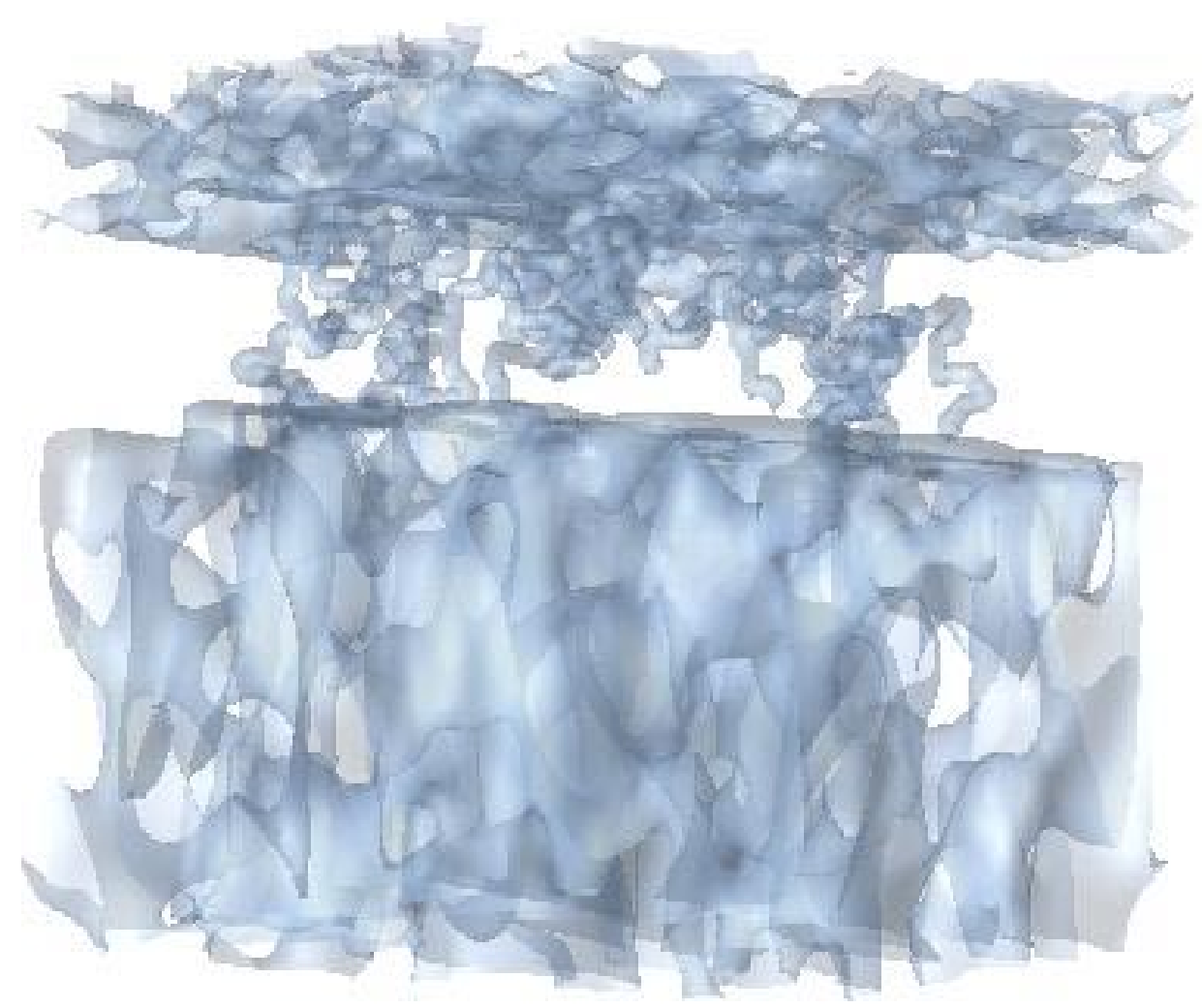

Fig. 3. Structure of the entire experimental domain (Fig. 1): Iso-surfaces of the mean log-scaling factors in the $A_{1}$ and $B$ horizon, together with the modelled structure of macropores in the $A_{2}$ horizon.

Table 1. Hydraulic parameters for the different structural units. For the $A_{1}$ and the $B$ horizons they were directly measured, for the $A_{2}$ and the macropores we used a plausible guess. For $n<2$, the parameter $h_{e}$ is significant for the air entry point instead of parameter $\alpha$ as indicated by brackets.

\begin{tabular}{lccccccc}
\hline & $\begin{array}{c}\alpha \\
{\left[\mathrm{cm}^{-1}\right]}\end{array}$ & $\begin{array}{c}n \\
{[-]}\end{array}$ & $\begin{array}{c}\theta_{S} \\
{\left[\mathrm{~cm}^{3} / \mathrm{cm}^{3}\right]}\end{array}$ & $\begin{array}{c}\theta_{r} \\
{\left[\mathrm{~cm}^{3} / \mathrm{cm}^{3}\right]}\end{array}$ & $\begin{array}{c}K_{s} \\
{[\mathrm{~cm} / \mathrm{h}]}\end{array}$ & $\begin{array}{c}\tau \\
{[-]}\end{array}$ & $\begin{array}{c}h_{e} \\
{[\mathrm{~cm}]}\end{array}$ \\
\hline$A_{1}$ horizon & $(33.0)$ & 1.24 & 0.34 & 0.1 & 18.0 & 0.5 & -10.2 \\
$A_{2}$ horizon & $(0.15)$ & 1.33 & 0.36 & 0.1 & 0.29 & 0.5 & -44.7 \\
macropores & 0.2 & 8.0 & 0.5 & 0.0 & 3600 & 0.5 & $(-0.58)$ \\
$B$ horizon & $(0.15)$ & 1.33 & 0.36 & 0.1 & 2.88 & 0.5 & -10.3 \\
\hline
\end{tabular}

multi-step outflow experiments and using the modified van Genuchten model as described above (Table 1). The parameters $h_{e}$ and $\alpha$ are both related to the air entry value and, depending on the parameter $n$, only one of them is actually relevant. The other one is put in brackets in Table 1). No direct measurements were available for the compacted $A_{2}$ horizon. The texture of this horizon was comparable to the horizons above and below. To account for the increased compactness of this layer we assumed a lower hydraulic conductivity and a larger air entry value. For the macropores we chose a value of $\alpha=0.2 \mathrm{~cm}^{-1}$. This corresponds to the air entry value of cylindrical pores of some $0.5 \mathrm{~mm}$ in diameter which is smaller than the actual size of our modelled macropores. This choice was motivated by the fact that macropores in soil are no perfect cylinders with smooth walls. As an additional positiv effect this choice increased the speed of the numerical solver. It has to be noted, however, that the parameter $\alpha$ determines the critical water potential at which the macropores start to conduct water. For our experiment this choice was not relevant, because the potential within the $A_{2}$ horizon was $\leq 0$ at the given water flux. The saturated hydraulic conductivity of the macropores was calculated from Poiseuille's law and a pore diameter of $0.5 \mathrm{~mm}$. The value of $n=8$ is high enough to make sure that the hydraulic conductivity of macropores 
drops quickly as soon as the water potential is below the air entry value to avoid artifacts in the dry range. The hydraulic parameters for all structural units are given in Table 1 .

The numerical simulations were conducted with a model using a cell-centred finite-volume scheme with fullupwinding in space and an implicit Euler scheme in time. The linear size of the discrete volume elements was equal to the radius of the macropores, i.e. $7.5 \mathrm{~mm}$. Linearisation of the nonlinear equations is done by an incomplete NewtonMethod with line search. The linear equations are solved with an algebraic multi-grid solver. For the time solver the time step is adapted automatically. A constant flux was imposed at the upper boundary of the domain and no-flux boundary conditions where used at the side boundaries. As there was no influence of a ground water table at this site, gravity flow was assumed at the lower boundary. After reaching a quasi-steady-state the flux field was interpolated using RT0 Raviart Thomas elements (Raviart and Thomas, 1975; Brezzi and Fortin, 1991).

Once the steady state velocity field was calculated, we simulated the infiltration of the dye tracer assuming a local convection-diffusion model. As the spatial heterogeneity of the soil was already considered explicitly in the calculation of the flux field, the effective diffusion coefficient $D_{0}$ was calculated as $D_{0}=\left[\theta^{7 / 3} / \phi^{2}\right] D_{m}$ using the second model of Millington and Quirk (1960) to account for the changes in water content, where $D_{m}$ is the molecular diffusion coefficient. The potential nonlinear sorption of brilliant blue was represented by a Langmuir isotherm $C_{s}^{\infty} \frac{k \theta C_{w}}{1+k \theta C_{w}}$. As the coefficients $k$ and $C_{s}^{\infty}$ are unknown for this soil we used similar values as obtained by Kasteel et al. (2002) in classical batch experiments which was $0.006 \mathrm{~m}^{3} / \mathrm{g}$ and $1.2 \mathrm{~g} / \mathrm{l}$, respectively. This is only thought as an orientation since the analysis of Kasteel et al. were made for another soil. Moreover, we assume that the sorption characteristics in macropores and matrix are the same which might not be the case in reality. The solute transport was discretized using a second-order Godonov method (LeVeque, 2002) with a minmod slope limiter for the convective part and a finite-volume scheme for the diffusive term.

The quasi-steady-state flux field was computed with a resolution of $128 \times 128 \times 128$ on a Pentium-4 Linux workstation, the solute transport was solved with a resolution of $256 \times 256 \times 256$ in parallel on the Linux cluster Helix (with 128 dual processor nodes). To speed up the calculation of the flux field we first calculated the quasi-steady state flux field with a resolution of $64 \times 64 \times 64$ starting with an initial condition of gravity flow and used the result as initial condition of the $128 \times 128 \times 128$ flux field.

The simulated time was the same as the duration of the experiment until the infiltration was stopped and the excavation started. We did not consider the subsequent period required to prepare all 24 serial sections. There was no marked difference in depth distribution of the tracer between the first and the last serial section, so that a direct comparison of the sim- ulated tracer distribution and the experimental data should be possible.

To evidence the effect of the mesoscopic heterogeneity, the sorption of the solute and the boundary conditions we simulated four different scenarios:

- $S C_{\text {reference }}$ a reference scenario including mesoscopic heterogeneity and sorption of the solute,

- $S C_{\text {no-sorp }}$ the same as $S C_{\text {reference }}$ but without sorption,

- $S C_{\text {homogeneous the same as } S C_{\text {reference }} \text { but without }}$ mesoscopic heterogeneity,

- $S C_{\text {low-flow }}$ the same as $S C_{\text {reference }}$ but with a flow rate of $0.13 \mathrm{~cm} / \mathrm{h}$ which is one order of magnitude lower than the rate in our experiment.

\section{Results and discussion}

The measured 3-D distribution of dye after the experiment in comparison to the simulation ( $S C_{\text {reference }}$ scenario) is illustrated in Fig. 4. For the simulation an iso-surface with a concentration of $0.1 \mathrm{~g} / \mathrm{l}$ is plotted. This threshold was chosen according to the analysis of Forrer et al. (2000) who found a clear visibility of brilliant blue above this value. This choice is expected to depend on the type of soil; however, the measured dye distribution patterns do not change significantly if we threshold at a slightly different value. The resolution of the experimental data is limited in one horizontal direction due to the separation of the serial sections of $4 \mathrm{~cm}$.

The simulated distribution of brilliant blue is much smoother than the measurements. However, it qualitatively reflects one striking characteristics of the measured data, i.e. the reduced dye coverage within the compacted part of the soil profile which increases again in the lower $B$ horizon. This pattern is an obvious consequence of the hydraulic structure of the soil (Table 1) especially of the fact that the saturated conductivity of the compacted $A_{2}$ horizon is lower than the infiltration rate. Consequently, most of the water is directed towards the macropores to bypass the compacted layer. For this configuration the absolute value of the saturated conductivity and the other hydraulic parameters of the $A_{2}$ horizons are of minor importance because the soil matrix is close to saturation. So the uncertainty of these parameters as a consequence of the estimation procedure is less critical for our experiment. On the other hand the ratio between $K_{S}$ and the flow rate triggers the incidence of preferential flow along the worm holes as dicussed further below.

For a more quantitative comparison of the different scenarios and the experiment with respect to the vertical distribution, the brilliant blue coverage was averaged over the horizontal cross section of the experimental domain. The resulting depth distributions are shown in Fig. 5 together with 

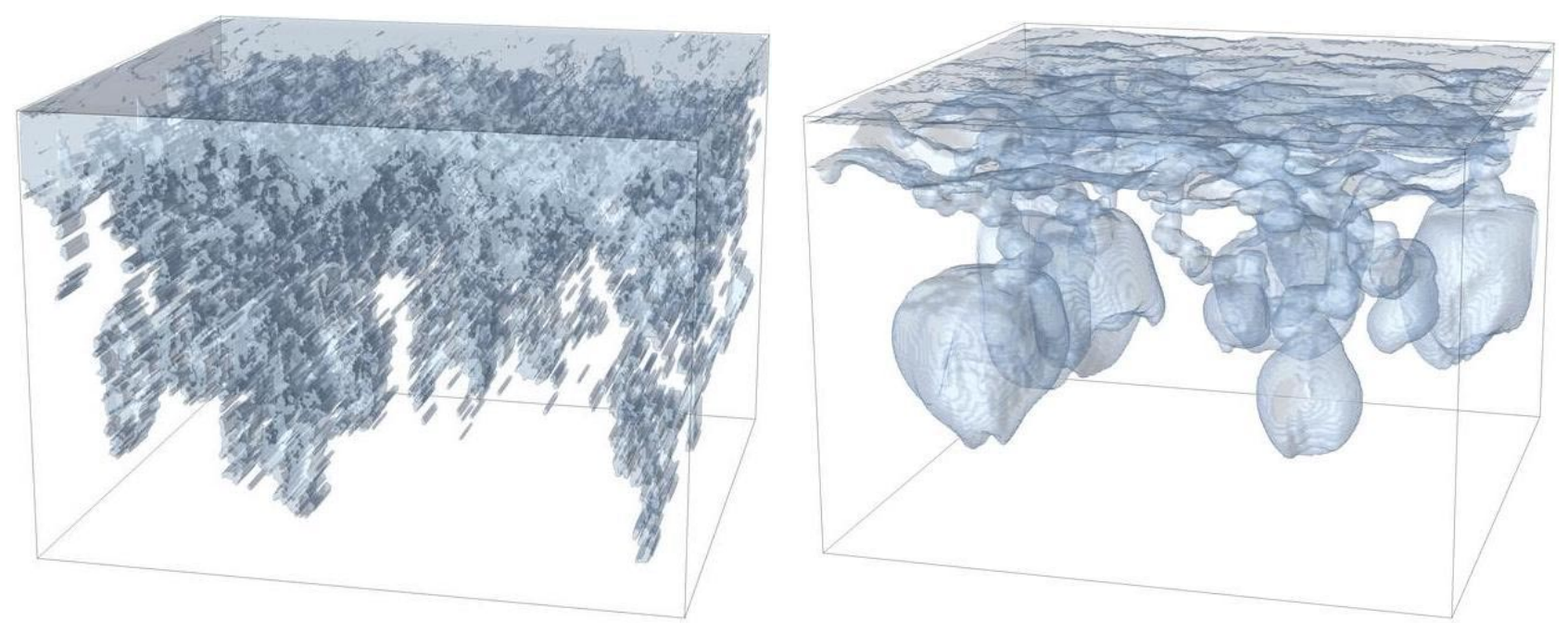

Fig. 4. Distribution of Brilliant Blue measured after the experiment (left) and simulated based on the heterogeneous structure of hydraulic properties (right) for the same initial and boundary conditions. The images cover the entire experimental domain shown in Fig. 1.
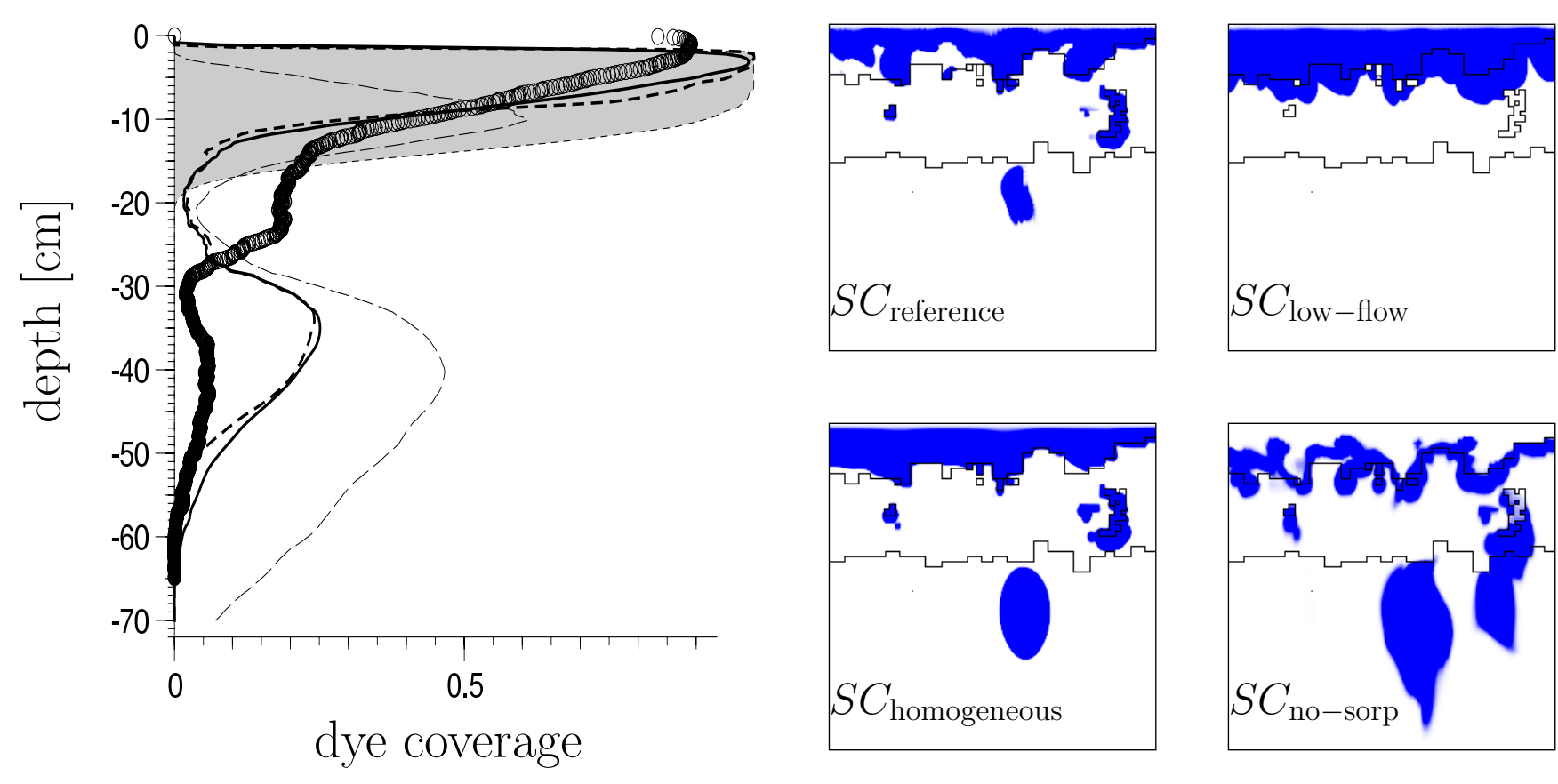

Fig. 5. Averaged vertical distribution of Brilliant Blue coverage measured after the experiment (symbols), simulated based on the heterogeneous structure of hydraulic properties including sorption $\left(S C_{\text {reference }}\right.$ thick line), without mesoscopic heterogeneities $\left(S C_{\text {homogeneous }}\right.$ thick dashed line), without sorption $\left(S C_{\text {no-sorp }}\right.$ dashed line) and with the low flow rate but same amount of infiltrated water ( $S C_{\text {low-flow }}$ grey). Notice that dye coverage is not necessarily related to total mass of dye. Right: vertical sections through the centre of the simulated domain are shown including the boundaries between the different materials (horizons and earthworm burrows). To compare with a measured section see Fig. 1. 
two-dimensional vertical sections showing the coverage of brilliant blue for the different scenarios.

The predicted depth distribution overestimates the amount of solute in the lower $B$ horizon and conversely, the dye concentration in the compacted $A_{2}$ horizon is underestimated. Obviously, more dye moved from the worm holes into the surrounding matrix. This is clearly due to the over simplification of the compacted horizon which is partitioned into a dense matrix and open macropores. Although, this roughly reflects the most relevant hydraulic structure of the material, the partitioning is more fuzzy in reality.

The overall pattern of transport is probably not sensitive to the detailed morphology of the macropores which were modelled to mimic the shape of earthworm burrows. A number of straight channels would have produced a similar pattern of solute transport in our experiment. But the more detailed morphology of macropores may be relevant for other processes where the interaction with the solid phase is stronger and hence, the surface density of macropores is more important. We think that the genetic approach used to represent the macropores is especially attractive in case the structure itself is difficult to measure directly. The example of earthworm burrows presented here is thought as a demonstration of this concept.

The effect of the mesoscopic heterogeneity is actually not visible in the overall phenomenology of solute transport, since the difference between $S C_{\text {homogeneous and } S C_{\text {reference }}}$ is negligible. In contrast, as expected, the depth distribution is significantly affected by the non-linear sorption of brilliant blue. For $S C_{\text {no-sorp }}$ the vertical translocation of brilliant blue is markedly increased while the overall pattern of solute distribution is not changed.

The scenario with the low flow rate shows a completely different behaviour. During $S C_{\text {low-flow }}$ the saturated conductivity of the compacted $A_{2}$ horizon is well above the simulated flow rate and consequently, the water potential remains well below water saturation. Under such conditions the conductivity of the macropores vanishes and the flow regime turns into a homogeneous matrix flow. The $S C_{\text {low-flow }}$ scenario has the same cumulative infiltration as the other scenarios; however, the mean vertical translocation of brilliant blue is much smaller compared to the scenarios with higher flow rate. This difference is mainly due to the absent macropore flow but enhanced by the non-linear sorption characteristics of the dye. Although a corresponding experiment is missing, $S C_{\text {low-flow }}$ highlights the predictive potential of the approach to model solute transport based on the structure of hydraulic properties. Using this approach, predictions for arbitrary initial and boundary conditions are generally possible. This is an advantage compared to the common approach of fitting an effective transport model to measured tracer distributions. The price we have to pay is the need of additional information on the structural composition of the material including the hydraulic properties of the structural components. On the other hand, in case of our experiment, a dual-porosity model calibrated to a break-through curve of the $A 2$ horizon would have reproduced the same general behavior with preferential flow at high flux and homogeneous transport at low flux. Hence, in this typical case, where macropores are embedded in a compacted matrix, dual-porosity models are much more efficient as long as conservative tracers are considered, because the structure of the material corresponds to the conceptual idea of the model. However we think that our approach is more general, since it is not focused on a special type of structure and all required parameters can be measured directly. It might thus also perform better in the case of reactive transport, when the spatial distribution of the solute is relevant for the reaction rates.

\section{Conclusions}

In this study we aimed at predicting the vertical translocation of a dye tracer in a heterogeneous field soil. The prediction was based on the representation of the complete threedimensional structure of the hydraulic properties and the resulting velocity field which was calculated using Richards' equation. To represent the structure we used the direct measurement of soil horizons, a statistical approach to estimate the sub-scale fluctuations of hydraulic properties and a genetic approach to model the formation of macroporous originating from earthworm activity.

The agreement between our experimental findings and the prediction of tracer distribution is far from beeing perfect. However, the overall pattern including the infiltration depth is reasonably matched. Given the fact, that the prediction is based on a only rough approximation of the structure and the related material properties we come to the following hypothesis which potentially could be corroborated by future experiments:

- Based on the structure of hydraulic properties, the approximate pattern of solute transport including the phenomenon of preferential flow can be predicted. The macroscopic dispersion is an immediate consequence of the macroscopic structure of the material. Given this structure, no "effective" dispersion coefficient is required.

- Given the relevant and continuous structure of the material, the hydraulic properties of the single structural units may either be measured directly (here: measurements of $h(\theta)$ and $K(\theta)$ for the different horizons), or they may be derived from proxy variables (here: estimated correlation length of bulk density within the horizons), or even derived from a plausible guess (here: $h(\theta)$ and $K(\theta)$ for earthworm burrows).

- To predict transport in heterogeneous soil, which cannot be considered to be macroscopically homogeneous, quantitative information on structure including 
the three-dimensional topology and on the related hydraulic properties is indispensable. If both components are available a rough approximation might be sufficient (Vogel and Roth, 2003). If one component is missing, the estimation of transport parameters is futile.

- To represent the structure of a model domain, different approaches such as direct measurements, statistical modelling, and modelling of structure formation can be combined. The latter is especially attractive for structural components that are hardly accessible by any measurement technique and it opens the possibility to make use of the knowledge available from other disciplines in soil science.

Acknowledgements. We are grateful to P. Berché, S. Lehmann, C. Le Lay, L. Raison and G. Yart for technical assistance during the field experiment. We would like to thank B. Labaye and A. Ternedde for data processing and image analysis and K. Roth for inspiring discussions. This work was partially funded by the "Agence Pour l'Accueil des Personnalites Etrangères" (A.P.A.P.E) and the "Deutscher Akademische Auslandsdienst" (DAAD) within a PROCOPE-project.

Edited by: W. Durner

\section{References}

Bastardie, F., Cannavacciuolo, M., Capowiez, Y., De-Dreuzy, J.-R., Bellido, A., and Cluzeau, D.: A new simulation for modelling the topology of earthworm burrow systems and their effects on macropore flow in experimental soils, Biol. Fert. Soils., 36, 161169, 2002.

Baveye, P. and Boast, C.: Fractal geometry, fragmentation processes and the physics of scale-invariance: An introduction, in: Fractals in Soil Science, edited by: Baveye, P., Parlange, J., and Stewart, B., Advances in Soil Science, CRC Press, Boca Raton, 1-54, 1998.

Beven, K. and Germann, P.: Macropores and water flow in soils, Water Resources Res., 18, 1311-1325, 1982.

Bouma, J.: Soil morphology and preferential flow along macropores, Agricultural Water management, 3, 235-250, 1981.

Brezzi, F. and Fortin, M.: Mixed and Hybrid Finite Element Methods, Springer-Verlag, New-York, 1991.

Capowiez, Y.: Differences in burrowing behaviour and spatial interaction between the two earthworm species Aporrectodea nocturna and Allolobophora chlorotica, Biol. Fert. Soils., 30, 310316, 2000.

Clothier, B. E., Heng, L., Magesan, G. N., and Vogeler, I.: The measured mobile-water content of an unsaturated soil as a function of hydraulic regime, Aust. J. Soil Res., 33, 397-414, 1995.

Cushman, J. H.: An introduction to hierarchical porous media, in: Dynamics of Fluids in Hierarchical Porous Media, edited by: Cushman, J. H., Academic Press, London, 1-6, 1990.

Dagan, G.: Statistical theory of groundwater flow and transport: Pore to laboratory, laboratory to formation and formation to regional scale, Water Resour. Res., 22, 120-134, 1986.

Flury, M. and Flühler, H.: Tracer characteristics of Brilliant Blue, Soil Sci. Soc. Am. J., 59, 22-27, 1995.
Flury, M., Flühler, H., Jury, W. A., and Leuenberger, J.: Susceptibility of soils to preferential flow of water: A field study, Water Resources Res., 30, 1945-1954, 1994.

Forrer, I., Papritz, A., Kasteel, R., Flühler, H., and Luca, D.: Quantifying dye tracers in soil profiles by image processing, Europ. J. Soil Sci., 51, 313-322, 2000.

Gelhar, L. W.: Stochastic subsurface hydrology. From theory to applications, Water Resour. Res., 22, 135-145, 1986.

Gerke, H. H. and van Genuchten, M. T.: A dual-porosity model for simulating the preferential movement of water and solutes in structured porous media, Water Resour. Res., 29, 305-319, 1993.

Germàn-Heins, J. and Flury, M.: Sorption of Brilliant Blue FCF in soils as affected by $\mathrm{pH}$ and ionic strength, Geoderma, 97, 87$101,2000$.

Hopmans, J. W., Cislerova, M., and Vogel, T.: X-ray tomography of soil properties, in: Tomography of Soil-Water-Root Processes, edited by: Ande, S. H. and Hopmans, J. W., ASA, SSSA Madison, Wisconsin, USA, 17-28, 1994.

Hopmans, J. W., Simunek, J., Romano, N., and Durner, W.: Methods of Soil analysis. Part 4, chap. Simultaneous determination of water transmission and retention properties. Inverse methods, SSSA, Madison, WI, 2002.

Ippisch, O., Vogel, H.-J., and Bastian, P.: Validity limits for the van genuchten-mualem model and implications for parameter estimation and numerical simulation, Adv. Water Res., in press, 2006.

Ippisch, O., Vogel, H.-J., and Bastian, P.: On the Necessity of an Entry Pressure in the Mualem Model, Tech. rep., University of Heidelberg, 2005.

Jarvis, N. J.: The MACRO model (Version 3.1.). Technical description and sample simulations, Tech. rep., Department of Soil Science, Swedish University of Agricultural Science, 1994.

Jarvis, N. J., Leeds-Harrison, P. B., and Dosser, J. M.: The use of tension infiltrometers to assess routes and rates of infiltration in a clay soil, J. Soil Sci., 38, 633-640, 1987.

Jegou, D., Hallaire, V., Cluzeau, D., and Trehen, P.: Characterization of the burrow system of the earthworms Lumbricus terrestris and Aporrectodea giardi using X-ray computed tomography and image analysis, Biology and Fertility of Soils, 29, 314318, 1999.

Kätterer, T., Schmied, B., Abbaspour, K. C., and Schulin, R.: Single- and dual-porosity modelling of multiple tracer transport through soil columns: effects of initial moisture and mode of application, Europ. J. Soil Sci., 52, 25-36, 2001.

Kasteel, R., Vogel, H., and Roth, K.: Effect of non-linear adsorption on the transport behaviour of Brilliant Blue in a field soil, Europ. J. Soil Sci., 53, 231-240, 2002.

Ketelsen, H. and Meyer-Windel, S.: Adsorption of brilliant blue FCF by soil, Geoderma, 90, 131-145, 1999.

Kowalsky, M. B., Finsterle, S., and Rubin, Y.: Estimating flow parameter distributions using ground-penetrating radar and hydrological measurements during transient flow in the vadose zone, Adv. Water Res., 27, 583-599, 2004.

Larsbo, M., Roulier, S., Stenemo, F., Kasteel, R., and Jarvis, N.: An improved dual-permeability model of water flow and solute transport in the vadose zone, Vadose Zone Journal, 4, 398-406, 2005.

LeVeque, R. J.: Finite Volume Methods for Hyperbolic Problems, Cambridge Texts in Applied Mathematics, Cambridge Univer- 
sity Press, Cambridge, 2002.

Lighthart, T. N., Peek, G. J., and Taber, E. J.: A method for the three-dimensional mapping of earthworm burrow systems, Geoderma, 57, 129-141, 1993.

Miller, E. E. and Miller, R. D.: Physical theory for capillary flow phenomena, J. Appl. Phys., 27, 324-332, 1956.

Millington, R. J. and Quirk, J. P.: Transport in porous media, 7th Intern. Congress of Soil Sci., Madison, Wisc., USA, 97-106, 1960.

Mualem, Y.: A new model for predicting the hydraulic conductivity of unsaturated porous media, Water Resour. Res., 12, 513-522, 1976.

Ould Mohammed, S. and Bruand, A.: Morphology, location in voids and origin of secondary calcite in soils from Beauce (France), in: Soil Morphology: Studies in Management and Genesis, edited by: Ringrose-Voase, A. J. and Humphreys, G., Elsevier, Amsterdam, 27-36, 1994.

Puvance, D. T. and Andricevic, R.: Geoelectric characterization of the hydraulic conductivity field and its spatial structure at variable scales, Water Resour. Res., 36, 2915-2924, 2000.

Raviart, P. A. and Thomas, J. M.: A Mixed Finite Element Method for 2-nd Order Elliptic Problems, in: Mathematical Aspects of Finite Element Methods, edited by: Dold, A. and Eckmann, B., Springer, lecture Notes of Mathematics, Volume 606, 1975.

Robin, M. J. L., Gutjahr, A. L., Sudicky, E. A., and Wilson, J. L.: Cross-correlated random field generation with the direct Fourier transform method, Water Resour. Res., 29, 2385-2397, 1993.

Roth, K.: Steady state flow in an unsaturated, two-dimensional, macroscopically homogeneous, Miller-similar medium, Water Resour. Res., 31, 2127-2140, 1995.
Stauffer, D.: Introduction to percolation theory, Taylor and Francis, London, 124 pp., 1985.

Sveistrup, T. E., Haraldsen, T. K., and Engelstad, F.: Earthworm channels in cultivated clayey and loamy Norwegian soils, Soil Till. Res., 43, 251-262, 1997.

van Genuchten, M. T.: A closed-form equation for predicting the hydraulic conductivity of unsaturated soils, Soil Sci. Soc. Am. J., 44, 892-898, 1980.

van Genuchten, M. T. and Wierenga, P. J.: Mass transfer studies in sorbing porous media. I. Analytical solutions, Soil Sci. Soc. Am. J., 40, 473-480, 1976.

Vanderborght, J., Vanclooster, M., Timmerman, A., Seuntjens, P., Mallants, D., Kim, D.-J., Jacques, D., Hubrechts, L., Gonzalez, C., Feyen, J., Diels, J., and Deckers, J.: Overview of inert tracer experiments in key Belgian soil types: Relation between transport and soil morphological and hydraulic properties, Water Resour. Res., 37, 2873-2888, 2001.

Vogel, H. J. and Roth, K.: Moving through scales of flow and transport in soil, J. Hydrol., 272, 95-106, 2003.

Vogel, T., van Genuchten, M. T., and Cislerova, M.: Effect of the shape of the soil hydraulic functions near saturation on variablysaturated flow predictions, Adv. Water Res., 24, 133-144, 2001.

Wildenschild, D., Hopmans, J. W., Vaz, C. M. P., Rivers, M. L., Rikard, D., and Christensen, B. S. B.: Using X-ray computed tomography in hydrology: Systems, resolutions, and limitations, J. Hydrol., 267, 285-297, 2002. 